\title{
The Mark Coventry, MD, Award: Oral Antibiotics Reduce Reinfection After Two-Stage Exchange: A Multicenter, Randomized Controlled Trial
}

\author{
Jonathan M. Frank MD, Erdan Kayupov MSE, Mario Moric MS, \\ John Segreti MD, Erik Hansen MD, Curtis Hartman MD, Kamil Okroj BS, \\ Katherine Belden MD, Brian Roslund PharmD, Randi Silibovsky MD, \\ Javad Parvizi MD, Craig J. Della Valle MD, \\ The Knee Society Research Group
}

Published online: 7 July 2016

(C) The Association of Bone and Joint Surgeons $\mathbb{R} 2016$

\begin{abstract}
Background Many patients develop recurrent periprosthetic joint infection after two-stage exchange arthroplasty of the hip or knee. One potential but insufficiently tested strategy to decrease the risk of persistent or recurrent infection is to administer additional antibiotics after the second-stage reimplantation.

Questions/purposes (1) Does a 3-month course of oral antibiotics decrease the risk of failure secondary to
\end{abstract}

The Knee Society Research Group: Scott M. Sporer MD, Department of Orthopaedic Surgery, Rush University Medical Center, Chicago, IL, USA, and Department of Orthopaedic Surgery, Central Dupage Hospital, Winfield, IL, USA; Matthew S. Austin MD, Department of Surgery, Rothman Institute at Thomas Jefferson University, Philadelphia, PA, USA; Kevin L. Garvin MD, University of Nebraska Medical Center, Omaha, NE, USA; Thomas Vail MD, University of California at San Francisco Medical Center, San Francisco, CA, USA; David Backstein MD, Department of Surgery, University of Toronto Medical Center, Toronto, Ontario, Canada; Timothy Tan MD, Department of Surgery, Rothman Institute at Thomas Jefferson University, Philadelphia, PA, USA; David Dalury MD, The St Joseph Medical Center, Towson, MD, USA; Michael A. Mont MD, Center for Joint Preservation and Replacement, Rubin Institute for Advanced Orthopedics, Sinai Hospital of Baltimore, Baltimore, MD, USA.

Each author certifies that he or she, or a member of his or her immediate family, has no funding or commercial associations (eg, consultancies, stock ownership, equity interest, patent/licensing arrangements, etc) that might pose a conflict of interest in connection with the submitted article.

All ICMJE Conflict of Interest Forms for authors and Clinical Orthopaedics and Related Research ${ }^{\mathbb{R}}$ editors and board members are on file with the publication and can be viewed on request.

Each author certifies that his or her institution approved the human protocol for this investigation, that all investigations were conducted in conformity with ethical principles of research, and that informed consent for participation in the study was obtained

This work was performed at the following institutions: Rush University Medical Center, Chicago, IL, USA: University of infection after a two-stage exchange? (2) Are there any complications related to the administration of oral antibiotics after a two-stage exchange? (3) In those patients who develop a reinfection, is the infecting organism different from the initial infection?

Methods Patients at seven centers randomized to receive 3 months of oral antibiotics or no further antibiotic treatment after operative cultures after the second-stage reimplantation were negative. Adult patients undergoing

California at San Francisco Medical Center, San Francisco, CA, USA; University of Nebraska Medical Center, Omaha, NE, USA; The Rothman Institute, Philadelphia, PA, USA; University of Toronto Medical Center, Toronto, Ontario, Canada; Towson Orthopaedic Associates, Towson, MD, USA; Central DuPage Hospital, Winfield, IL, USA; and The Rubin Institute, Baltimore, MD, USA.

\section{J. M. Frank}

Steadman-Philippon Research Institute, Vail, CO, USA

E. Kayupov, M. Moric, J. Segreti, K. Okroj,

C. J. Della Valle (ه)

Department of Orthopaedic Surgery, Rush University Medical

Center, 1725 West Harrison Street, Suite 300, Chicago,

IL 60612, USA

e-mail: craig.dellavalle@rushortho.com

\section{E. Hansen}

University of California at San Francisco Medical Center, San Francisco, CA, USA

C. Hartman

University of Nebraska Medical Center, Omaha, NE, USA

K. Belden, B. Roslund, R. Silibovsky, J. Parvizi

The Rothman Institute at Thomas Jefferson University Hospital, Philadelphia, PA, USA 
two-stage hip or knee revision arthroplasty for a periprosthetic infection who met Musculoskeletal Infection Society (MSIS) criteria for infection at the first stage were included. Oral antibiotic therapy was tailored to the original infecting organism(s) in consultation with an infectious disease specialist. MSIS criteria as used by the treating surgeon defined failure. Surveillance of patients for complications, including reinfection, occurred at 3 weeks, 6 weeks, 3 months, 12 months, and 24 months. If an organism demonstrated the same antibiotic sensitivities as the original organism, it was considered the same organism; no DNA subtyping was performed. Analysis was performed as intent to treat with all randomized patients included in the groups to which they were randomized. A log-rank survival curve was used to analyze the primary outcome of reinfection. At planned interim analysis (enrollment is ongoing), 59 patients were successfully randomized to the antibiotic group and 48 patients to the control group. Fiftyseven patients had an infection after TKA and 50 after a THA. There was no minimum followup for inclusion in this analysis. The mean followup was 14 months in the antibiotic group and 10 months in the control group.

Results Patients treated with oral antibiotics failed secondary to infection less frequently than those not treated with antibiotics (5\% [three of 59] versus 19\% [nine of 48]; hazard ratio, 4.37; 95\% confidence interval, 1.297-19.748; $\mathrm{p}=0.016$ ). Three patients had an adverse reaction to the oral antibiotics severe enough to cause them to stop taking the antibiotics early, and four patients who were randomized to that group did not take the antibiotics as directed. With the numbers available, there were no differences between the study groups in terms of the likelihood that an infection after treatment would be with a new organism (eight of nine in the control group versus one of three in the treatment group, $\mathrm{p}=0.087$ ).

Conclusions This multicenter randomized trial suggests that at short-term followup, the addition of 3 months of oral antibiotics appeared to improve infection-free survival. As a planned interim analysis, however, these results may change as the study reaches closure and the safety profile may yet prove risky. Further followup of this cohort of patients will be necessary to determine whether these preliminary results are durable over time.

Level of Evidence Level I, therapeutic study.

\section{Introduction}

Periprosthetic joint infections (PJIs) remain one of the most devastating complications of THA and TKA, posing a large physical, economic, and emotional burden on the patient and many challenges on the surgeon. Although there are several strategies for the treatment of PJI, a commonly used strategy to treat patients with late infection is a twostage revision with placement of an antibiotic-impregnated spacer and a course of organism-directed antibiotics after the first stage with reported success rates approaching $90 \%$ [4]. However, more recent studies have suggested that failures secondary to infection occur more frequently than that [2-4].

In a retrospective study by Zywiel et al. [14], a course of microorganism-directed oral antibiotic therapy after reimplantation reduced the risk of recurrent infection after a two-stage exchange. Similarly, Johnson et al. [5] found a $13.6 \%$ rate of reinfection compared with $0 \%$ for those patients treated with a course of oral antibiotics. However, to date, there have been no randomized studies evaluating this strategy.

We therefore asked: (1) Does a 3-month course of oral antibiotics decrease the risk of recurrent infection after a two-stage exchange? (2) Are there any complications related to the administration of oral antibiotics after a twostage exchange? (3) In those patients who develop a reinfection, is the infecting organism different from the initial infection?

\section{Patients and Methods}

This was a multicenter, prospective randomized controlled trial. Each participating site received approval from their respective institutional review boards. This study was registered with clinicaltrials.gov (NCT01760863). All adult patients presenting to the participating institutions since 2011 who met eligibility criteria were approached for enrollment and enrollment is ongoing. A planned interim analysis was done once approximately half of the sample size was enrolled to determine if a large difference was observed and if the study should be halted. After discussion of the results with the institutional review board at Rush University Medical Center and with our statistician, the decision was made to continue enrolling.

Adult patients undergoing two-stage revision arthroplasty of the knee or hip for a periprosthetic infection who met Musculoskeletal Infection Society (MSIS) criteria for infection [10] at the first stage and who had negative cultures at the time of the second-stage reimplantation were included. Exclusion criteria included patients with fungal prosthetic joint infections, unable to tolerate or refuse to undergo oral antibiotic therapy, unable to followup at the specified intervals, allergy to the therapy of preference, or had an infecting organism that was not amenable to oral antibiotic therapy.

Patients were randomized through opaque envelopes prepared with a random number generator to either a 3month course of oral antibiotic therapy tailored to the 
original infecting organism(s) or no additional antibiotic therapy.

Patients were evaluated at the 3-week, 6-week, 3-month, 12-month, and 24-month time points. At each followup visit, patients who were randomized to the oral antibiotic arm were evaluated for any missing doses, additional prescribed antibiotics, adverse reactions, and reinfection. Patients randomized to the observation group were evaluated for any additional prescribed antibiotics and reinfection at each followup visit.

The primary outcome measure was reinfection as determined by meeting MSIS criteria as ascertained by the treating surgeon. Assessors were not blinded relative to the treatment groups. Patients were evaluated for complications at the time of last followup. If an organism demonstrated the same antibiotic sensitivities as the original organism, it was considered the same organism; no DNA subtyping was performed.

\section{Statistical Analysis}

An a priori power analysis determined that 77 patients per group would be required to demonstrate a reduction in infection recurrence from $16 \%$ to $4 \%(\beta=0.80$ and $\alpha=$ 0.05). Analysis was performed as intent to treat (ITT) with all randomized patients included in the groups to which they were randomized. A log-rank survival curve was used to analyze the primary outcome of reinfection, and the time to last followup or date of reinfection was used; $\mathrm{p}$ values $<$ 0.05 were considered significant. The data presented here were generated at the time of an interim analysis once half of the target sample size patients were enrolled.

There was a total of 57 patients with PJI after a TKA and 50 patients with a PJI after THA. Mean age, body mass index, gender, and Charlson Comorbidity Index were not different between the groups suggesting appropriate randomization. No minimum followup period was set and loss to followup was not ascertained.

A total of 107 patients were enrolled at the time of interim analysis with 58 patients randomized to the antibiotic group and 49 in the control group. Time to followup tended to be longer in the treatment group than in the control group $(14 \pm 11$ months versus $10 \pm 10$ months; $\mathrm{p}<0.0526$; Table 1). This was largely related to patients in the control group failing more frequently, decreasing the mean time to followup. A minimum followup period was not set for the current interim analysis. There was one screening failure in which a patient randomized to the oral antibiotic group was found to be allergic to the only oral antibiotic regimen recommended. Forty-eight patients in the control group and 59 patients in the oral antibiotic group remained eligible for followup and were included in this analysis.

\section{Results}

Failure from infection occurred less commonly in the treatment group than the control group. Three patients in the treatment group were diagnosed with infection compared with nine in the control group (5\% versus 19\%; hazard ratio, 4.37; 95\% confidence interval, 1.297-19.748; $\mathrm{p}=0.0162$; Fig. 1). Ten of the 12 reinfections occurred before 1 year, and of those, two were from the antibiotic group and eight from the control group. Of the two reinfections that occurred after 1 year, one was from the antibiotic group and one was from the control group (Table 2).

Three patients stopped taking their assigned oral antibiotics early because they developed adverse reactions including gastrointestinal upset and nausea. Three additional patients in the oral antibiotic group reported milder adverse reactions including rash, yeast infection, and nausea, but all three completed their assigned courses. Patients were not removed from the ITT analysis as a result of premature discontinuation of assigned antibiotic treatment.

With the numbers available, there were no differences between the study groups in terms of the likelihood that an infection after treatment would be with a new organism (eight of nine in the control group versus one of three in the treatment group, $\mathrm{p}=0.087$ ).

Table 1. Preoperative demographic characteristics of both the oral antibiotics and control groups

\begin{tabular}{|c|c|c|c|c|c|c|c|}
\hline \multirow[t]{2}{*}{ Demographic characteristics } & \multicolumn{2}{|c|}{ Oral antibiotics } & \multicolumn{2}{|l|}{ Control } & \multirow[b]{2}{*}{$\mathrm{p}$ value } & \multicolumn{2}{|c|}{ All data } \\
\hline & Mean & $(\mathrm{SD})$ & Mean & $(\mathrm{SD})$ & & Mean & (SD) \\
\hline Age (years) & 63 & $(10)$ & 65 & (11) & 0 & 64 & $(10)$ \\
\hline $\mathrm{BMI}\left(\mathrm{kg} / \mathrm{m}^{2}\right)$ & 31 & (7) & 31 & (6) & 1 & 31 & (6) \\
\hline Gender & 33 male & (56\% male) & 33 male & (69\% male) & & & \\
\hline Charlson Comorbidity Index & 1 & (1) & 1 & (1) & 1 & 1 & (1) \\
\hline Time to followup (months) & 14 & $(11)$ & 10 & $(10)$ & 0 & 12 & $(11)$ \\
\hline
\end{tabular}

Continuous data are shown as mean $\pm \mathrm{SD}$; $\mathrm{BMI}=$ body mass index. 


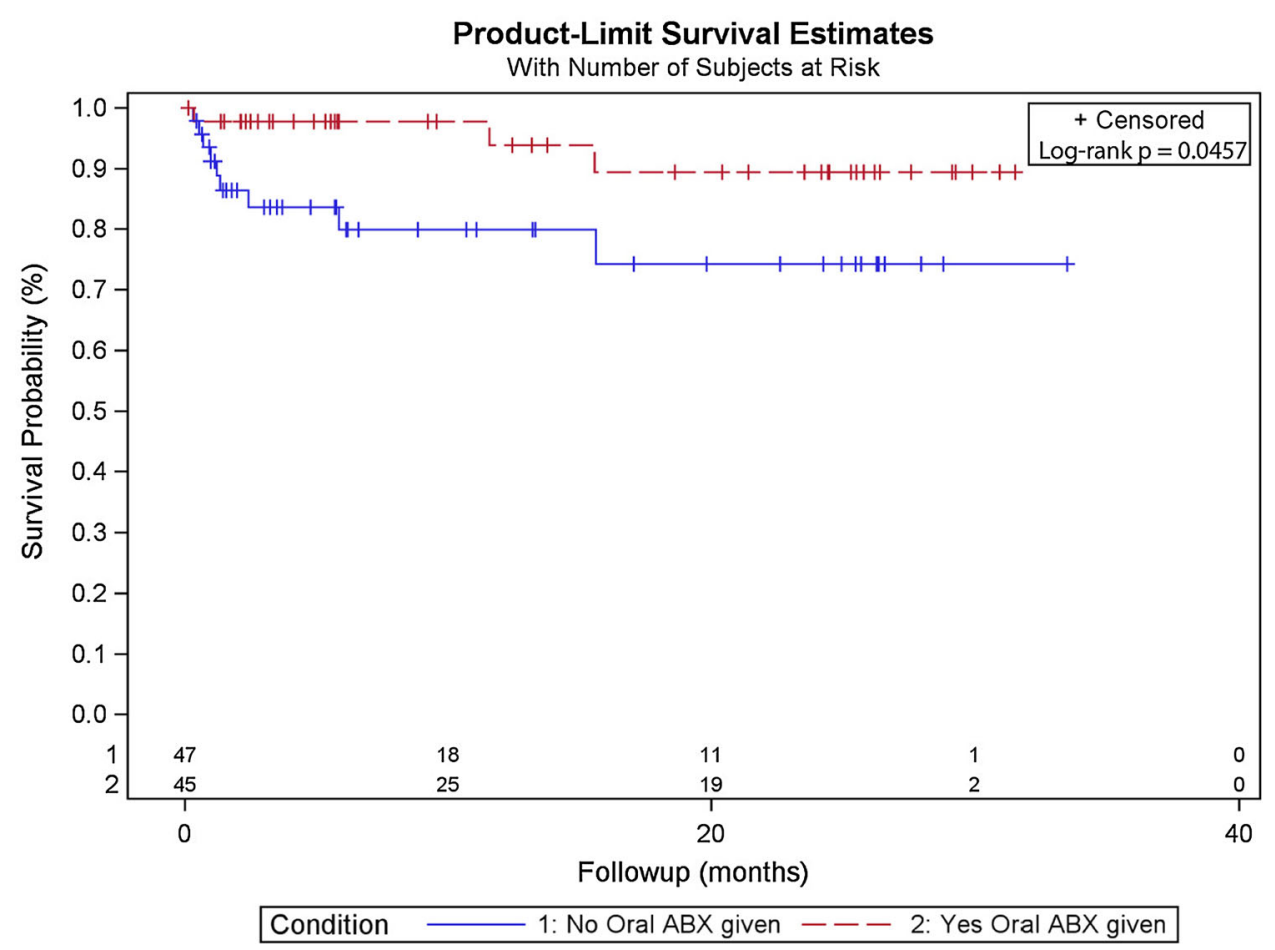

Fig. 1 A log-rank survival curve for oral antibiotics versus control for all spacers used is shown. ABX = antibiotics

\section{Discussion}

Treatment of PJI continues to be challenging. Treatment failures are not only costly, but also are associated with substantial morbidity and mortality $[1,3,6,8,12]$ Although two-stage exchange arthroplasty remains a commonly used treatment approach, it remains imperfect. Studies have reported success rates approaching 90\%, whereas others have reported less successful outcomes [7]. The patient devastation that ensues with failure has necessitated the push for continued attempts at improving outcomes. Prior retrospective studies [5, 14] have shown promising improvements in infection-free rates with the incorporation of oral antibiotics after two-stage exchange. Unfortunately, their low patient numbers and retrospective design weaken the strength of these studies. Therefore, in the current study, we performed a multicenter randomized control trial that investigated the utility of an additional 3month course of oral antibiotics after a two-stage exchange for PJI. At interim analysis, we have found a significant decrease in the rate of reinfection from 19\% in the control group to $5 \%$ in the treatment group.

There are several limitations to this study that should be considered when interpreting our results. Given this is an interim analysis, there was a relatively small number of patients that has been enrolled at this time and we have not yet reached our target enrollment. However, insofar as we identified between-group differences that are potentially clinically important at our interim analysis, we felt it was important to share our findings. There is also the potential for assessor bias given this study was randomized yet not blinded. Following strict MSIS criteria for reinfection helped decreased this. Furthermore, our followup is relatively short and it will be critical to understand in time if the results in the oral antibiotic group are durable over time. That being said, it has been suggested that the majority of infections observed in this population occurred within the first 3 months postoperatively [9]. The patients included in this study were also recruited from a variety of institutions that may introduce cluster bias; however, the demographics were not different among groups and we believe including patients from multiple centers makes our work more generalizable. In addition, culture sensitivities were used to determine if an infecting organism was "different" from the original infecting organism; however, genotyping may have shown that an organism may be different although demonstrating the same sensitivity, thus providing additional evidence that a failure secondary to reinfection was a new infection versus a persistent infection. The clinical relevance of this, however, may be small given that the treatment algorithm would remain unchanged.

It is important to recognize that this is an interim analysis and that this introduces the potential for serious limitations to our findings. This interim analysis was planned once approximately half of the patients were 
Table 2. Culture results from subjects with a reinfection

\begin{tabular}{|c|c|c|c|}
\hline Joint & $\begin{array}{l}\text { Time to reinfection status } \\
\text { post-Stage } 2 \text { (days) }\end{array}$ & Treatment & Culture results \\
\hline Hip & 9 & Control & $\begin{array}{l}\text { 1. Stage } 1 \text { culture: Enterococcus faecalis Group D } \\
\text { 2. Reinfection culture: coagulase-negative Staphylococcus }\end{array}$ \\
\hline Knee & 10 & ABX: levofloxacin & $\begin{array}{l}\text { 1. Stage } 1 \text { culture: } E \text { coli } \\
\text { 2. Reinfection culture: MRSA }\end{array}$ \\
\hline Hip & 16 & Control & $\begin{array}{l}\text { 1. Stage } 1 \text { Culture: anaerobic Gram-positive cocci } \\
\text { 2. Reinfection culture: coagulase-negative staphylococcus }\end{array}$ \\
\hline Hip & 21 & Control & $\begin{array}{l}\text { 1. Stage } 1 \text { culture: Staphylococcus epidermidis, MSSA } \\
\text { 2. Reinfection culture: MSSA }\end{array}$ \\
\hline Knee & 29 & Control & $\begin{array}{l}\text { 1. Stage } 1 \text { Culture: MSSA } \\
\text { Reinfection culture: Serratia marcescens }\end{array}$ \\
\hline Hip & 36 & Control & $\begin{array}{l}\text { 1. Stage } 1 \text { culture: MSSA } \\
\text { 2. Reinfection culture: Escherichia coli, MSSA }\end{array}$ \\
\hline Hip & 40 & Control & $\begin{array}{l}\text { 1. Stage } 1 \text { culture: MSSA } \\
\text { 2. Reinfection culture: Streptococcus agalactiae (Group B) }\end{array}$ \\
\hline Hip & 72 & Control & $\begin{array}{l}\text { 1. Stage } 1 \text { culture: MSSA } \\
\text { 2. Reinfection culture: coagulase-negative Staphylococcus }\end{array}$ \\
\hline Hip & 175 & Control & $\begin{array}{l}\text { 1. Stage } 1 \text { culture: Proteus mirabilis } \\
\text { 2. Reinfection culture: Morganella morganii }\end{array}$ \\
\hline Knee & 347 & ABX: cefadroxil & $\begin{array}{l}\text { 1. Stage } 1 \text { Culture: coagulase-negative Staphylococcus, } \\
\text { Mycoplasma avium complex } \\
\text { 2. Reinfection culture: Staphylococcus epidermidis }\end{array}$ \\
\hline Knee & 467 & ABX: cefuroxime & $\begin{array}{l}\text { 1. Stage } 1 \text { culture: MSSA } \\
\text { 2. Reinfection culture: MSSA }\end{array}$ \\
\hline Knee & 468 & Control & $\begin{array}{l}\text { 1. Stage } 1 \text { culture: Bacterioides fragilis } \\
\text { 2. Reinfection culture: Staphylococcus lugdunensis }\end{array}$ \\
\hline
\end{tabular}

$\mathrm{ABX}=$ antibiotics; $\mathrm{MSSA}=$ methicillin-susceptible Staphylococcus aureus; MRSA = methicillin-resistant $S$ aureus.

enrolled to ensure that there was not a large difference between the two groups. Once we identified a difference that we felt was potentially clinically relevant (a reduction in the failure rate secondary to infection from $19 \%$ to $5 \%$ ), we felt it was important to share our findings. Once this analysis was performed, we discussed with our institutional review board if the study should be halted, and after meeting with the institutional review board and our statistician, the decision was made to continue because a few more failures in the oral antibiotic group would change the results; this is a serious limitation of our work and mandates future followup at longer term followup to ensure that these results do not change. Based on our a priori power calculation, target enrollment is 154 patients followed for a minimum of 2 years.

We found that oral antibiotics resulted in a decreased risk of infection at short-term followup. Several studies have focused on the use of oral antibiotics as a method of chronic suppression after two-stage revision surgery to maintain improved survival rates. Byren et al. [2] noted a decrease in the risk of reinfection for patients who were kept on oral antibiotics during the first 3 months after surgery in comparison to those patients who ceased treatment. More recently, Siquiera et al. [11] found a 5-year infection-free survival rate that was almost $20 \%$ higher for those patients who received chronic oral antibiotic suppression versus those who did not. Two retrospective studies have also reported a decreased risk of infection with the addition of a course of oral antibiotic therapy after reimplantation. Zywiel et al. [14] found the risk of infection after treatment with oral antibiotics was $4 \%$ compared with $16 \%$ without oral antibiotics, whereas Johnson et al. [5] found a $0 \%$ rate of reinfection versus $13.6 \%$ without antibiotic treatment. The results of our randomized trial seem to support the work of these prior retrospective series, although further followup over time will be required. It is possible that oral antibiotic treatment after the second-stage reimplantation may only delay recurrent infection and not change the overall reinfection rate with further followup.

Although the administration of oral antibiotics is a relatively low-risk intervention, it is important to recognize that antibiotic administration is not without risk. In this series, three patients (just more than 5\%) had to discontinue their use secondary to complications related to their 
administration. Furthermore, many patients may not recognize the importance of taking them and discontinue them early even if recommended by the surgeon or another caretaker. It is also important to keep in mind that oral antibiotics add cost to patient treatment. Finally, longer term antibiotic treatment could promote antibiotic resistance, which is a growing concern.

Interestingly, a large proportion of the reinfections in this study was caused by a new organism, suggesting that a course of antibiotics may help protect patients from reinfection with a new microorganism as opposed to further treating the infection that originally led to the two-stage exchange. Prior studies have also suggested that most cases of recurrent PJI may be in fact secondary to a new infection rather than a failure of the prior treatment [13].

This multicenter randomized trial suggests that at shortterm followup, the addition of 3 months of oral antibiotics appeared to improve infection-free survival. As a planned interim analysis, however, these results may change as the study reaches closure and the safety profile may yet prove risky. Further followup of this cohort of patients will be necessary to determine whether these preliminary results are durable over time.

Acknowledgments The authors would like to acknowledge Dr. Paul Yi, Dr. Matthew Tetrault, Dr. Nicholas Brown, Dr. Nathan Wetters, Judy Guerreiro, Stephanie Miller and Dr. Vinay Aggarwal for assistance with the collection of data related to this study. Further, the authors wish to thank Dr. Antonia Chen for assistance with data collection and the enrollment of patients into the study and Dr. James Purtill and Dr. Greg Deirmengian for the enrollment of patients into the study.

\section{References}

1. Bozic KJ, Ries MD. The impact of infection after total hip arthroplasty on hospital and surgeon resource utilization. $J$ Bone Joint Surg Am. 2005;87:1746-1751.

2. Byren I, Bejon P, Atkins BL, Angus B, Masters S, McLardySmith P, Gundle R, Berendt A. One hundred and twelve infected arthroplasties treated with 'DAIR' (débridement, antibiotics and implant retention): antibiotic duration and outcome. J Antimicrob Chemother. 2009;63:1264-1271.

3. Darouiche RO. Treatment of infections associated with surgical implants. N Engl J Med. 2004;350:1422-1429.

4. Hanssen AD. Managing the infected knee: as good as it gets. $J$ Arthroplasty. 2002;17:98-101.

5. Johnson AJ, Zywiel MG, Jones LC, Delanois RE, Stroh DA, Mont MA. Reduced re-infection rates with postoperative oral antibiotics after two-stage revision hip arthroplasty. BMC Musculoskelet Disord. 2013;14:123.

6. Klouche S, Sariali E, Mamoudy P. Total hip arthroplasty revision due to infection: a cost analysis approach. Orthop Traumatol Surg Res. 2010;96:124-132.

7. Kurd MF, Ghanem E, Steinbrecher J, Parvizi J. Two-stage exchange knee arthroplasty: does resistance of the infecting organism influence the outcome? Clin Orthop Relat Res. 2010;468:2060-2066.

8. Kurtz SM, Lau E, Schmier J, Ong KL, Zhao K, Parvizi J. Infection burden for hip and knee arthroplasty in the United States. J Arthroplasty. 2008;23:984-991.

9. Liu C, Kakis A, Nichols A, Ries MD, Vail TP, Bozic KJ. Targeted use of vancomycin as perioperative prophylaxis reduces periprosthetic joint infection in revision TKA. Clin Orthop Relat Res. 2014;472:227-231.

10. Parvizi J, Zmistowski B, Berbari EF, Bauer TW, Springer BD, Valle Della CJ, Garvin KL, Mont MA, Wongworawat MD, Zalavras CG. New definition for periprosthetic joint infection: from the Workgroup of the Musculoskeletal Infection Society. Clin Orthop Relat Res. 2011;469:2992-2994.

11. Siqueira MBP, Saleh A, Klika AK, O'Rourke C, Schmitt S, Higuera CA, Barsoum WK. Chronic suppression of periprosthetic joint infections with oral antibiotics increases infection-free survivorship. J Bone Joint Surg Am. 2015;97:1220-1232.

12. Zmistowski B, Karam JA, Durinka JB, Casper DS, Parvizi J. Periprosthetic joint infection increases the risk of one-year mortality. J Bone Joint Surg Am. 2013;95:2177-2184.

13. Zmistowski B, Tetreault MW, Alijanipour P, Chen AF, Valle Della CJ, Parvizi J. Recurrent periprosthetic joint infection: persistent or new infection? J Arthroplasty. 2013;28:1486-1489.

14. Zywiel MG, Johnson AJ, Stroh DA, Martin J, Marker DR, Mont MA. Prophylactic oral antibiotics reduce reinfection rates following two-stage revision total knee arthroplasty. Int Orthop. 2011;35:37. 